EPJ Web of Conferences 75, 04007 (2014)

DOI: 10.1051/epjconf/ 20147504007

(C) Owned by the authors, published by EDP Sciences, 2014

\title{
Iron oxide nanocomposite magnets produced by partial reduction of strontium hexaferrite
}

\author{
Jussi Tikkanen ${ }^{1,2, a}$ and Petriina Paturi ${ }^{1}$ \\ ${ }^{1}$ Wihuri Physical Laboratory, Department of Physics and Astronomy, University of Turku, FI-20014, Finland \\ ${ }^{2}$ University of Turku Graduate School (UTUGS), University of Turku, FI-20014, Finland
}

\begin{abstract}
Isotropic bulk nanocomposite permanent magnets were produced with strontium hexaferrite, $\mathrm{SrO} \cdot 6 \mathrm{Fe}_{2} \mathrm{O}_{3}$, and magnetite, $\mathrm{Fe}_{3} \mathrm{O}_{4}$, as the magnetically hard and soft components. A novels synthesis scheme based on the partial reduction of $\mathrm{SrO} \cdot 6 \mathrm{Fe}_{2} \mathrm{O}_{3}$ was employed. In two parallel experiments, nano- and microcrystalline $\mathrm{SrO} \cdot 6 \mathrm{Fe}_{2} \mathrm{O}_{3}$ particles were compacted into pellets along with a controlled, understoichiometric amount of potato starch as a reducing agent. The pellets were then sintered in a passive atmosphere. Based on $\mathrm{XRD}$ and room temperature magnetic hysteresis measurements, it was concluded that a fraction of the $\mathrm{SrO} \cdot 6 \mathrm{Fe}_{2} \mathrm{O}_{3}$ input material had been reduced into $\mathrm{Fe}_{3} \mathrm{O}_{4}$. In comparison with pure $\mathrm{SrO} \cdot 6 \mathrm{Fe}_{2} \mathrm{O}_{3}$ control pellets, these composites exhibited maximum energy product increases in excess of $5 \%$ due to remanence boosting. The improvement of magnetic properties was attributed to an efficient exchange spring coupling between the magnetic phases. Interestingly, as the synthesis scheme also worked for microcrystalline $\mathrm{SrO} \cdot 6 \mathrm{Fe}_{2} \mathrm{O}_{3}$, the method could presumably be adapted to yield crystallographically oriented bulk nanocomposite magnets.
\end{abstract}

\section{Introduction}

Despite the impressive performance characteristics of rare-earth permanent magnets, the considerably cheaper magnetoplumbite (M) ferrites barium hexaferrite, $\mathrm{BaO} \cdot 6 \mathrm{Fe}_{2} \mathrm{O}_{3}$, and strontium hexaferrite, $\mathrm{SrO} \cdot 6 \mathrm{Fe}_{2} \mathrm{O}_{3}$ remain the hard magnetic materials of choice for a majority of everyday applications. A great deal of research has been targeted on the improvement of these magnetic derivatives of iron(III) oxide, and although some progress has been made in terms of maximum energy products, $B H_{\max }$, by introducing suitable dopant chemicals like La and $\mathrm{Co}$, it seems that the conventional evolution of ferrite magnets is approaching its practical boundaries [1].

One novel way to improve upon the properties of Mferrites would be to combine them heterogeneously with other magnetic materials with useful complementary properties, to produce composite permanent magnets. In this paper, work regarding the rather natural combination of strontium hexaferrite, $\mathrm{SrO} \cdot 6 \mathrm{Fe}_{2} \mathrm{O}_{3}$, and magnetite, $\mathrm{Fe}_{3} \mathrm{O}_{4}$, a magnetically softer ferrite, is reported. Whereas $\mathrm{SrO} \cdot 6 \mathrm{Fe}_{2} \mathrm{O}_{3}$ is renowed for its high magnetic anisotropy $\left(K_{1}=350 \mathrm{~kJ} \mathrm{~m}^{-3}\right.$ [2]) but is somewhat short on saturation magnetization $\left(M_{\mathrm{s}}=380 \mathrm{kA} \mathrm{m}^{-1}[2]\right), \mathrm{Fe}_{3} \mathrm{O}_{4}$ would bring in more magnetization $\left(M_{\mathrm{s}}=480 \mathrm{kA} \mathrm{m}^{-1}\right.$ [2]) provided that some external mechanism could be utilized to boost its nearly nonexistent coercivity sufficiently to retain a high remanence, a centrally important property for any permanent magnet [2].

\footnotetext{
${ }^{\mathrm{a}}$ Corresponding author: jussi.tikkanen@utu.fi
}

One such coercivity mechanism, the exchange spring, was theoretically described by Kneller and Hawig in 1991 [3]. To summarize, the mechanism requires a nanocomposite of the magnetically hard and soft phases to be constructed in such a manner that a maximal volume fraction of the soft component lies within the exchange length [2]

$$
l_{\mathrm{ex}}=\sqrt{\frac{A}{\mu_{0} M_{\mathrm{s}}^{2}}}
$$

of the hard component. Here $A$ is the exchange stiffness of the hard phase, roughly proportional to its Curie temperature. $\mu_{0}$ and $M_{\mathrm{s}}$ represent vacuum permeability and saturation magnetization, respectively. Once within the exchange length of a sufficiently anisotropic magnetic material, a soft magnetic material will find it energetically favorable to align its magnetization with the hard phase. The composite of the two will then function as a permanent magnet with a synergetically enhanced remanence and, ideally, almost the coercivity of the original hard magnetic phase [3]. For the M-ferrites, $l_{\mathrm{ex}}$ evaluates to about $6 \mathrm{~nm}$ [2].

Functioning hard-soft nanocomposite magnets have been realized in numerous experiments, e.g. [4, 5], but the particular combination of $\mathrm{Fe}_{3} \mathrm{O}_{4}$ and $\mathrm{M}$-ferrites has seen rather limited interest and success [6, 7]. An excellent review on the general subject of nanocomposite magnets can be found in [8]. 


\section{Experimental}

\subsection{Particle synthesis}

$\mathrm{SrO} \cdot 6 \mathrm{Fe}_{2} \mathrm{O}_{3}$ nanoparticles were synthesized using a solgel combustion method largely based on the work of $\mathrm{T}$. M. H. Dang et al. [9]. Unless otherwise stated, reagents of pro analysi grade were used at all times.

A 1:11:36 mole fraction mixture of the following ingredients was dissolved into water: strontium chloride hexahydrate, $\mathrm{SrCl}_{2} \cdot 6 \mathrm{H}_{2} \mathrm{O}$, iron(III) nitrate nonahydrate, $\mathrm{Fe}\left(\mathrm{NO}_{3}\right)_{3} \cdot 9 \mathrm{H}_{2} \mathrm{O}$, and citric acid monohydrate, $\mathrm{C}_{6} \mathrm{H}_{8} \mathrm{O}_{7} \cdot \mathrm{H}_{2} \mathrm{O}$. The solution was homogenized by stirring for one hour, after which a stoichiometric amount of aqueous ammonia solution was added to roughly neutralize the citric acid.

The neutralized solution was stirred for a few more minutes and oven-dried at $90{ }^{\circ} \mathrm{C}$ for several days, until a solid orange residue was obtained. The residue was transferred into a well ventilated furnace held at $500{ }^{\circ} \mathrm{C}$, where it ignited and was allowed to remain for $5 \mathrm{~h}$. The resulting gray fluff was annealed at $1050{ }^{\circ} \mathrm{C}$ for $2 \mathrm{~h}$ in air (heating ramp $1 \mathrm{~h}$ ), yielding a dark gray powder of nanocrystalline $\mathrm{SrO} \cdot 6 \mathrm{Fe}_{2} \mathrm{O}_{3}$. The furnace was allowed a 5-hour passive cooldown to room temperature before extracting the products.

Microcrystalline $\mathrm{SrO} \cdot 6 \mathrm{Fe}_{2} \mathrm{O}_{3}$ particles were produced by an adaptation of the solid-state diffusion method. Strontium nitrate, $\mathrm{Sr}\left(\mathrm{NO}_{3}\right)_{2}$, and microcrystalline hematite powder, $\alpha-\mathrm{Fe}_{2} \mathrm{O}_{3}$, were mixed with a few drops of water to dissolve the nitrate. The guideline $\mathrm{Sr}^{2+}: \mathrm{Fe}^{3+}$ molar ratio 1:11, optimized for nanoparticles [9], was again followed. The $\alpha-\mathrm{Fe}_{2} \mathrm{O}_{3}$ used was not of commercial pro analysi grade, but an industrial byproduct provided by Rautaruukki Oyj, Finland (XRD purity $100 \% \pm 1 \%$, grain size $440 \mathrm{~nm}$ ).

The wet mixture was annealed at $1200{ }^{\circ} \mathrm{C}$ for $2 \mathrm{~h}$ (heating ramp $1 \mathrm{~h}$ ) and given a 5-hour passive cooldown to room temperature. A dark gray batch of slightly fused $\mathrm{SrO} \cdot 6 \mathrm{Fe}_{2} \mathrm{O}_{3}$ aggregates was obtained.

A quality control of the $\mathrm{SrO} \cdot 6 \mathrm{Fe}_{2} \mathrm{O}_{3}$ and $\alpha-\mathrm{Fe}_{2} \mathrm{O}_{3}$ powders was performed by measuring their XRD diffractograms (Philips X'Pert Pro diffractometer, $\mathrm{Cu} \mathrm{K} \mathrm{\alpha}_{\text {. }}$ radiation) and magnetic hysteresis loops (Quantum Design PPMS, ACMS option) at $300 \mathrm{~K}$. A Rietveld refinement was performed using the Maud software [10]. Some essential figures of merit have been collected in table 1 for the $\mathrm{SrO} \cdot 6 \mathrm{Fe}_{2} \mathrm{O}_{3}$ powders. A demagnetizing factor $N=0.25$ was used when calculating the remanences, determined by measuring the slope of a soft ferromagnetic $\left(\mathrm{Fe}_{3} \mathrm{O}_{4}\right)$ hysteresis curve near zero external field. The magnetometer samples were rolled into ellipsoids of PTFE tape.

The microparticle synthesis scheme was not optimized to any great extent, and $11 \%$ of the particle mass was left as $\alpha-\mathrm{Fe}_{2} \mathrm{O}_{3}$. This was considered manageable, since the magnetic properties of $\alpha-\mathrm{Fe}_{2} \mathrm{O}_{3}$ at $300 \mathrm{~K}$ are well known [2] and were easily factored out during analysis based on XRD-determined mass fractions.
Table 1. Characteristic figures of $\mathrm{SrO} \cdot 6 \mathrm{Fe}_{2} \mathrm{O}_{3}$ precursor powders: nominal diameters $(d)$, coercivities of magnetization $\left(H_{\mathrm{c}}\right)$, remanent magnetizations $\left(M_{\mathrm{r}}\right)$ and purities by mass.

\begin{tabular}{|c|c|c|c|}
\hline $\boldsymbol{d}(\mathbf{n m})$ & $\boldsymbol{H}_{\mathbf{c}}\left(\mathrm{kA} \mathrm{m}^{-\mathbf{1}}\right)$ & $\boldsymbol{M}_{\mathbf{r}}\left(\mathrm{kA} \mathrm{m}^{-\mathbf{1}}\right)$ & Purity \\
\hline 35 & 370 & 185 & $100 \%$ \\
\hline 440 & 250 & 140 & $89 \%$ \\
\hline
\end{tabular}

\subsection{Sample preparation and measurements}

Two analogous series of prototype magnets, one with $\mathrm{SrO} \cdot 6 \mathrm{Fe}_{2} \mathrm{O}_{3}$ nanoparticles and one with microcrystalline particles, were manufactured by hydraulically dry-compacting the precursor powders $(20$ min at $30 \mathrm{MPa}$ ) and sintering the pellets in a tube furnace $\left(2 \mathrm{~h}\right.$ under $\mathrm{Ar}$ gas flow at $1050{ }^{\circ} \mathrm{C}, 1 \mathrm{~h}$ heating ramp, $5 \mathrm{~h}$ passive cooldown). In addition to the $\mathrm{SrO} \cdot 6 \mathrm{Fe}_{2} \mathrm{O}_{3}$ particles, a varying amount of foodstuff-grade potato starch, $\mathrm{C}_{27} \mathrm{H}_{48} \mathrm{O}_{20}$, was mixed into each sample prior to compaction. The starch was to function as a reductant and partially transform the $\mathrm{SrO} \cdot 6 \mathrm{Fe}_{2} \mathrm{O}_{3} \mathrm{M}$-ferrite phase into magnetically softer $\mathrm{Fe}_{3} \mathrm{O}_{4}$.

In summary, both the nanoparticle sample series and the microparticle series contained four pellets, with the amount of starch varying between $0 \%, 4 \%, 6 \%$ and $9 \%$ by mass. The mass fractions were only fixed to within $\pm 1 \%$. The chemical composition and magnetic properties of the pellets were verified by measuring their XRD powder diffractograms and magnetic hysteresis loops at $300 \mathrm{~K}$. Additionally, surface texture measurements were done with the diffractometer in an Eulerian cradle configuration. All samples were found to be crystallographically isotropic.

\section{Results}

The primary basis for evaluating a permanent magnet material lies in its intrinsic working temperature hysteresis loop, $M(H)$. As the signal given by the PPMS $A C M S$ magnetometer was in terms of effective magnetic moments, $\mu$, vs. the external field, $H_{\text {ext }}$, the samples were weighed on an analytical balance to the precision of 0.05 $\mathrm{mg}$ for converting the data into magnetizations, $M$, vs. internal fields, $H$. Sample masses were of order of $10 \mathrm{mg}$.

Considering their powderous nature, the density of the samples was approximated as that of bulk $\mathrm{SrO} \cdot 6 \mathrm{Fe}_{2} \mathrm{O}_{3}, \rho=5290 \mathrm{~kg} \mathrm{~m}^{-3}$, multiplied by the packing factor of a hcp structure, $\pi / \sqrt{18}$. Also, the approximate demagnetizing factor $N=0.25$ was taken into account. Thus, the total coversion function from measured effective magnetic moments to volume magnetizations took the form

$$
M=\frac{\pi \rho \mu}{\sqrt{18} m(1-N)}
$$

where $m$ is the sample mass. Knowing $M$, the internal field $H$ could be calculated from $H_{\text {ext }}$ and the demagnetizing field, $H_{\mathrm{d}} \approx-N M[2]$, as 
Table 2. Figures of merit for the prototype composite magnets: remanent magnetizations, $M_{\mathrm{r}}$, coercivities of magnetization, $H_{\mathrm{c}}$, and intrinsic maximum energy products, $\mathrm{BH}_{\max }$. Microparticle series corrected for XRD-determined $\alpha-\mathrm{Fe}_{2} \mathrm{O}_{3}$ content.

\begin{tabular}{|c|c|c|c|}
\hline Sample & $\begin{array}{c}\boldsymbol{H}_{\mathbf{c}} \\
\left(\mathbf{k A ~ m}^{-1}\right)\end{array}$ & $\begin{array}{c}\boldsymbol{M}_{\mathbf{r}} \\
\left(\mathbf{k A ~ m}^{-1}\right)\end{array}$ & $\begin{array}{c}\boldsymbol{B H}_{\mathbf{m a x}} \\
\left(\mathbf{k J ~ m}^{-\mathbf{3}}\right)\end{array}$ \\
\hline nano 0 \% & 311 & 191 & 9.7 \\
\hline nano 4\% & 266 & 200 & 10.4 \\
\hline nano 6\% & 9 & 123 & 0.5 \\
\hline nano 9\% & 4 & 35 & 0.0 \\
\hline micro 0 \% & 249 & 154 & 6.2 \\
\hline nano 4\% & 200 & 198 & 7.6 \\
\hline nano 6\% & 200 & 183 & 6.3 \\
\hline nano 9\% & 1 & 99 & 0.1 \\
\hline
\end{tabular}

$$
H=H_{\mathrm{ext}}+H_{\mathrm{d}} \approx H_{\mathrm{ext}}-N M
$$

The hysteresis loops measured from both the nanoparticle and microparticle sample series are presented in figure 1. The main figures of merit for each loop can be found in table 2. The $B H_{\max }$ values given have been calculated as the absolute maxima of the expression

$$
-B H=-\mu_{0}(H+M) H
$$

It can be seen that this form of the maximum energy product is, ideally, independent of sample shape, and thus a well-defined material parameter. The leading minus sign was included to give positive $B H_{\max }$ values, since the extrema in question are reached within the second and fourth quadrants of the hysteresis curves.

Both sample series exhibit a very similar response to the addition of the starch reductant. Remarkably, a modest starch addition was observed to coincide with an increase of the maximum energy product, $B H_{\max }$, in both cases $v s$. the $0 \%$ control sample.

With nanoparticles of $\mathrm{SrO} \cdot 6 \mathrm{Fe}_{2} \mathrm{O}_{3}$, adding $4 \%$ starch increased $B H_{\max } 7.2 \%$ from $9.7 \mathrm{~kJ} \mathrm{~m}^{-3}$ to $10.4 \mathrm{~kJ} \mathrm{~m}^{-3}$. The energy products were reached at reverse $H$-fields of $79.6 \mathrm{kA} \mathrm{m}^{-1}$ and $81.6 \mathrm{kA} \mathrm{m}^{-1}$, respectively. Looking at the corresponding hysteresis loops on the left side of figure 1 , it can be seen that a tradeoff between coercivity and remanence has taken place, but in a manner that has been favorable for the $\mathrm{BH}_{\max }$ of the starch-reduced sample. Unfortunately, the mass fraction of $\mathrm{Fe}_{3} \mathrm{O}_{4}$ responsible for the remanence boost was too low to be reliably determined via XRD and Rietveld refinement - it could only be estimated to be below $5 \%$.

When $6 \%$ starch was added, a very narrow loop was measured, with a prominent constriction around the origin. This curve form is typical of heterogeneous mixtures of magnetically soft and hard components that are poorly exchange coupled [3] - in essence, the linear superposition of two very different hysteresis curves is witnessed. A majority of the $\mathrm{SrO} \cdot 6 \mathrm{Fe}_{2} \mathrm{O}_{3}$ particles had been reduced into magnetically soft $\mathrm{Fe}_{3} \mathrm{O}_{4}$, most of which lay out of the reach of the hard-phase exchange field. Yet more pronounced signs of reductant excess were seen with the $9 \%$ sample. This time, a fraction of the $\mathrm{Fe}_{3} \mathrm{O}_{4}$ was apparently reduced even further into nonmagnetic wüstite, $\mathrm{FeO}$.

When the $\mathrm{SrO} \cdot 6 \mathrm{Fe}_{2} \mathrm{O}_{3}$ nanoparticles were exchanged for microcrystalline particles, a qualitatively similar series of hysteresis loops resulted. Also this time, the addition of $4 \%$ starch improved $B H_{\max }$ significantly, now by an impressive $22.6 \%$ from $6.2 \mathrm{~kJ} \mathrm{~m}^{-3}$ to $7.6 \mathrm{~kJ} \mathrm{~m}^{-3}$.

Based on the huge relative boost and the fact that the $4 \%$ loop's demagnetizing quadrants are just barely convex, one must conclude that the amount of reductant must have been quite optimal. A little bit more starch, and the magnetic phases would have decoupled, as in the $6 \%$ microparticle loop, where slight concave kinks can already be seen in the demagnetizing quadrants. Note, however, that the $6 \%$ sample still exceeded the $B H_{\max }$ of the $0 \%$ control. With $9 \%$ starch added, the microparticle series ended in a textbook soft magnetic $\mathrm{Fe}_{3} \mathrm{O}_{4}$ hysteresis curve with a minuscule coercivity and a saturation magnetization of order $400 \mathrm{kA} \mathrm{m}^{-1}$.

The absolute $B H_{\max }$ values of the microparticle samples were somewhat smaller than for the analogous nanoparticle composites, owing to the weaker coercivity of the microparticles. The $B H_{\max }$ values of the $0 \%$ and 4 $\%$ samples were reached at $H$-fields $61.9 \mathrm{kA} \mathrm{m}^{-1}$ and 59.5 $\mathrm{kA} \mathrm{m}^{-1}$, respectively.

\section{Discussion}

To roughly summarize the reduction of $\mathrm{SrO} \cdot 6 \mathrm{Fe}_{2} \mathrm{O}_{3}$ into $\mathrm{Fe}_{3} \mathrm{O}_{4}$ by potato starch, the following reaction equation can be proposed:

$$
\begin{aligned}
58 \mathrm{SrO} \cdot & 6 \mathrm{Fe}_{2} \mathrm{O}_{3}+3 \mathrm{C}_{27} \mathrm{H}_{48} \mathrm{O}_{20} \\
& \rightarrow 58 \mathrm{Sr}+232 \mathrm{Fe}_{3} \mathrm{O}_{4}+81 \mathrm{CO}_{2}+72 \mathrm{H}_{2} \mathrm{O}
\end{aligned}
$$

Apparently, some (liquid) strontium can be expected to be left as an impurity. One should note that $\mathrm{CO}$, a gaseous intermediate product and also a reductant, will greatly contribute to the dynamics of the reaction due to its easy diffusion. The reaction is also assumed to take place at the surfaces of solid $\mathrm{SrO} \cdot 6 \mathrm{Fe}_{2} \mathrm{O}_{3}$ crystals, making its kinetics very sensitive to particle morphology. Therefore, all in all, the reaction equation (5) can only be taken as a qualitative description of the composite synthesis process.

The fact that the composite is formed by transforming some $\mathrm{SrO} \cdot 6 \mathrm{Fe}_{2} \mathrm{O}_{3}$ into $\mathrm{Fe}_{3} \mathrm{O}_{4}$ in situ during sintering means that the hard-soft magnetic phase interface will be very clean, allowing efficient interphase exchange coupling. While it is extremely difficult to determine the optimal reductant amount theoretically, it is easy to construct an approximate $B H_{\max }$ prediction for these composite magnets, based on the Stoner-Wohlfarth model [2].

Let's consider a single spherical core-shell particle magnet, with an interior of $\mathrm{SrO} \cdot 6 \mathrm{Fe}_{2} \mathrm{O}_{3}$ and the topmost $10 \mathrm{~nm}$ reduced into $\mathrm{Fe}_{3} \mathrm{O}_{4}$. Although the soft phase layer thickness exceeds the exchange length $l_{\mathrm{ex}}=6 \mathrm{~nm}$ given in section 1, it is arguable that this amount of $\mathrm{Fe}_{3} \mathrm{O}_{4}$ could be efficiently exchange-coupled when a multitude of 

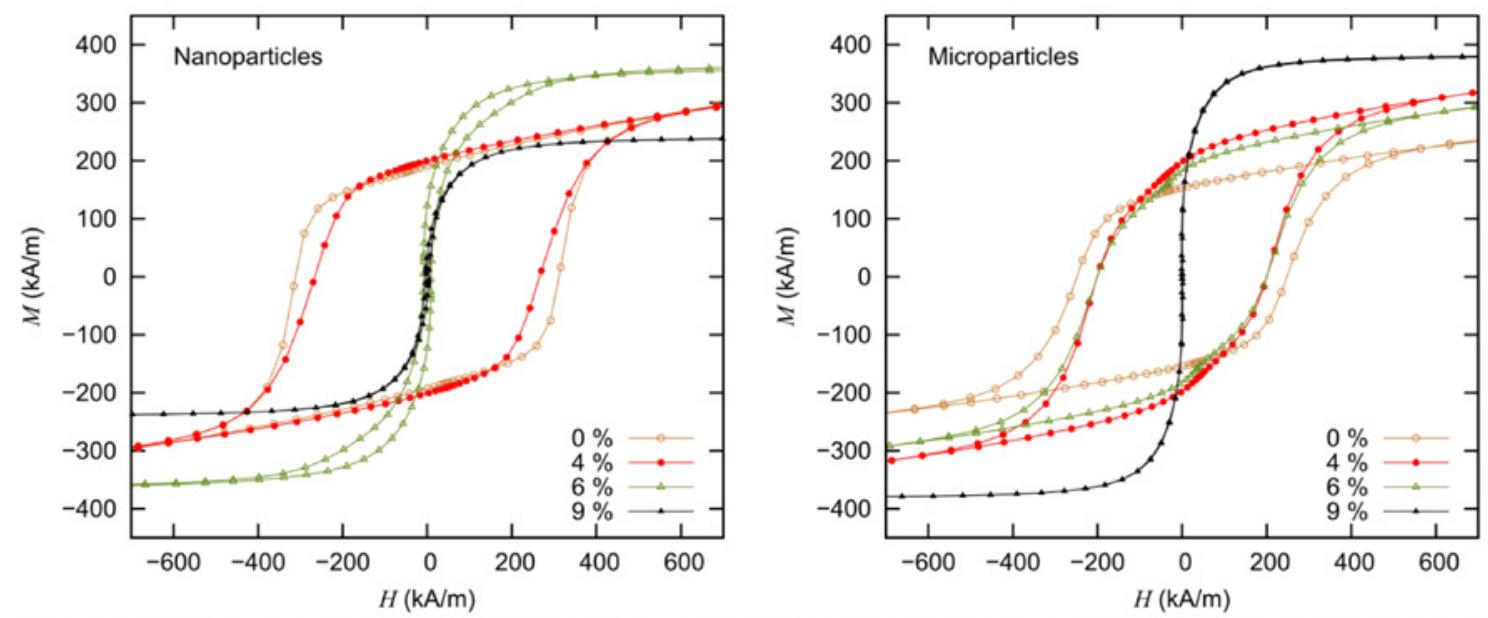

Figure 1. The $M(H)$ hysteresis curves of the nanoparticle series (left) and microparticle series (right), measured at $300 \mathrm{~K}$, with initial starch mass fractions indicated in $\%$. The microparticle curves have been corrected for XRD-determined $\alpha$ - $\mathrm{Fe}_{2} \mathrm{O}_{3}$ content.

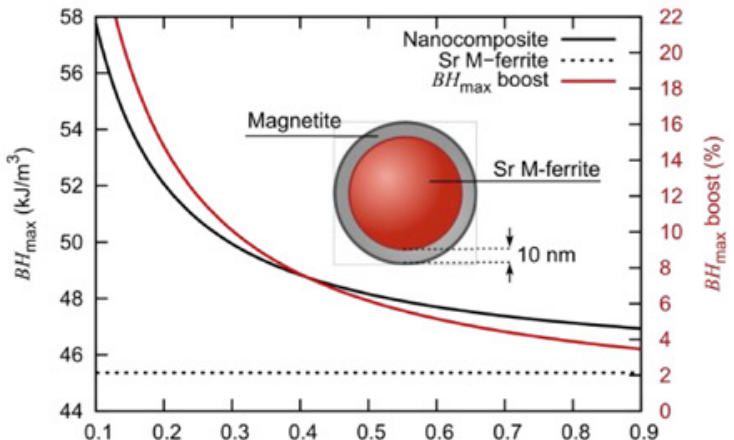

Figure 2. An ideal $B H_{\max }$ prediction for grain-oriented $\mathrm{SrO} \cdot 6 \mathrm{Fe}_{2} \mathrm{O}_{3} / \mathrm{Fe}_{3} \mathrm{O}_{4}$ core-shell composite magnets (sketch inset) vs. pure $\mathrm{SrO} \cdot 6 \mathrm{Fe}_{2} \mathrm{O}_{3} \mathrm{M}$-ferrite.

particles fuse together. Assuming sufficient coercivity and perfect crystal texture, the expression for $\mathrm{BH}_{\max }$ can be simplified to

$$
B H_{\max }=\frac{1}{4} \mu_{0} M_{\mathrm{r}}^{2}
$$

The easy-axis remanence of the composite magnet, $M_{\mathrm{r}}$, equal to its saturation magnetization in this "square-loop" model, can be approximated as the volume-weighted average of the component bulk magnetizations, $M_{\mathrm{r}}\left(\mathrm{SrO} \cdot 6 \mathrm{Fe}_{2} \mathrm{O}_{3}\right)=380 \mathrm{kA} \mathrm{m}{ }^{-1}$ and $M_{\mathrm{r}}\left(\mathrm{Fe}_{3} \mathrm{O}_{4}\right)=480 \mathrm{kA}$ $\mathrm{m}^{-1}$ (data from [2]). Thus, one can plot $\mathrm{BH}_{\max }$ from eq. (6) as a function of particle diameter, as in figure 2. The single-phase M-ferrite prediction $\left(B H_{\max }=45 \mathrm{~kJ} \mathrm{~m}^{-3}\right)$ agrees with published calculations [2]. Further numerical calculations for isotropic Stoner-Wohlfarth magnets suggested a practically identical relative boost curve.

\section{Conclusions}

Through a novel starch reduction method, isotropic hardsoft nanocomposite magnets were synthesized with strontium hexaferrite and magnetite as the hard and soft magnetic phases, respectively. The composites were found to yield maximum energy products that systematically exceeded those of pure $\mathrm{SrO} \cdot 6 \mathrm{Fe}_{2} \mathrm{O}_{3}$, regardless of the size of the $\mathrm{SrO} \cdot 6 \mathrm{Fe}_{2} \mathrm{O}_{3}$ particles used as precursors. Using microparticle precursors, the process could presumably be adapted to yield oriented bulk nanocomposite magnets, whose $B H_{\max }$ values would theoretically exceed those of pure $\mathrm{SrO} \cdot 6 \mathrm{Fe}_{2} \mathrm{O}_{3}$ by over $5 \%$. Promisingly, an experimental $B H_{\max }$ boost of $23 \%$ was observed with isotropic samples.

\section{Acknowledgements}

We acknowledge the Jenny and Antti Wihuri Foundation and Prizztech Oy, Finland, for financial support, and especially Martti Paju for valuable industry-specific insights.

\section{References}

1. K. H. J. Buschow (ed.), Concise Encyclopedia of Magnetic and Superconducting Materials, $2^{\text {nd }}$ ed., 175-183 (Elsevier, Amsterdam, 2005)

2. J. M. D. Coey, Magnetism and Magnetic Materials, 234-249, 418-427, 472 (Cambridge University Press, New York, 2009)

3. E. Kneller and R. Hawig, IEEE Transactions on Magnetics 27, 3588-3600 (1991)

4. H. Zeng et al., Nature 420, 395-398 (2008)

5. C. Rong et al., Applied Physics Letters 96, 102513 (2010)

6. P. Bercoff and H. Bertorello, Journal of Magnetism and Magnetic Materials 187, 169176 (1998)

7. M. Molaei et al., Journal of Superconductivity and Novel Magnetism 25, 519-524 (2012)

8. J. P. Liu et al., Nanoscale Magnetic Materials and Applications, $2^{\text {nd }}$ ed., 309-332 (Springer, Dordrecht, 2009)

9. T. M. H. Dang et al., Advances in Natural Sciences: Nanoscience and Nanotechnology 3, 025015 (2012)

10. Luca Lutterotti, Material Analysis Using Diffraction version 2.33 [computer program] (1997-2011) 